\title{
Linking reward expectation to behavior in the basal ganglia
}

\author{
Joshua I. Gold
}

Department of Neuroscience, University of Pennsylvania, 116 Johnson Pavilion, 3600 Hamilton Walk, Philadelphia, PA 19104-6074, USA

\begin{abstract}
Psychological factors such as expected reward value can have strong effects on behavior. Although signals related to reward have been found in numerous brain regions, how these signals are used by the circuits that control action is unknown. A recent study suggests that neurons in the caudate nucleus of the basal ganglia could play a role in transforming expected reward into a spatially selective behavioral bias.
\end{abstract}

Poor Paris was asked to make a decision that even Zeus had avoided. Who was the most beautiful goddess: Hera, Athena or Aphrodite? Fearful and uncertain about how his decision would be received by the losers, he tried tactfully but unsuccessfully - to declare a three-way tie. Told to make a decision, Paris received assurances that there would be no reprisals and, even better, was offered rewards by the goddesses to try to influence him. Hera promised that if he picked her, he would become the King of Asia, as well as the richest man alive. Athena promised that Paris would be victorious in all battles, and that he would become the most handsome and wise man in the world. Aphrodite offered, simply, the beautiful Helen of Troy. It was no contest: Aphrodite's reward easily biased Paris' decision in her favor.

The fact that 'psychological' factors, including uncertainty and anticipated costs and benefits, biased the judgment of Paris and can affect similarly subjective decisions is to be expected. Perhaps more surprising is that these factors can also shape our ability to perform even mundane sensory-motor tasks, influencing how well and how quickly we perceive sensory stimuli and execute appropriate motor commands [1,2]. However, as central as these influences are to both complex and simple behaviors, little is known about the underlying neural mechanisms. For example, despite a growing body of work identifying neural circuits that represent information related to reward in the context of sensory-motor tasks [3], how these kinds of signals might influence behavior is a mystery. A recent study by Lauwereyns and colleagues has begun to shed light on this difficult issue [4]. The results describe neural signals in a region of the basal ganglia called the caudate nucleus that appear to relate anticipation of an uncertain reward with the preparation for a goal-directed eye movement.

\section{Caudate responses correspond to an advance bias}

In their study, Lauwereyns and colleagues trained monkeys to perform a 'biased saccade task' (BST). On a given trial, the monkey fixated a central spot of light until a target (another spot of light) appeared to the left or to the right. Once the target appeared, the monkey made a saccadic eye movement to it as quickly as possible. Then came the crucial feature of the task: after correctly making the saccade, the monkey was rewarded (with a sound plus a drop of liquid) only if the target was in one of the two possible locations. The rewarded location was fixed in blocks of 20 consecutive trials, such that only correct leftward saccades were rewarded in one block, only correct rightward saccades were rewarded in the next block, and so on. This block-wise design meant that the monkey knew before the target appeared which location would lead to a reward but did not know in which location it would actually appear. Predictably, this asymmetrical reward schedule resulted in a predisposition to make an eye movement to the rewarded location, evident as shorter response times (the amount of time elapsed between the appearance of the target and the initiation of the eye movement) to that location.

To find the neural signals that might be responsible for generating these reward-related changes in response times, Lauwereyns and colleagues recorded activity in the caudate nucleus. As part of the basal ganglia, the caudate is thought to play a role in the control of movement and, in particular, appears to be involved in the generation of saccadic eye movements [5]. It is also one of several structures in the brain, along with other parts of the basal ganglia and several areas of cortex, that represent the anticipated availability or magnitude of a reward provided in the context of a behavioral task [3]. Indeed, several recent studies from Hikosaka's research group have indicated that caudate activity that anticipates a memory-guided saccade is sensitive to the contingency between the visual cue and the reward [6,7]. They designed the BST to address how this reward-related activity might be used in the process of generating the saccade.

The activity of individual caudate neurons during the BST reflected the asymmetrical reward schedule. After selecting only neurons that showed a reliable increase in activity in the 1.5 seconds preceding target onset, they found that in $\sim 75 \%$ of these neurons this increase tended to be associated with just one of the two position-reward contingencies. Specifically, 25 neurons responded more strongly when the rewarded target was in the hemifield contralateral to the recording site, whereas six neurons responded more strongly when the rewarded target was ipsilateral to the recording site. For the contralateralfavoring neurons, at least, the effect seemed to be robust, with differential activity appearing as much as 1.5 seconds 
before target onset and increasing in magnitude until the target appeared.

Thus, both the eye movements and the caudate responses reflected reward-related biases. When rightward saccades were rewarded, the monkey made rightward saccades more quickly than leftward saccades, and left (contralateral) caudate activity was high whereas right (ipsilateral) caudate activity was low. When leftward saccades were rewarded, the effects were reversed. Moreover, the rate at which these physiological and behavioral effects adapted to a change in the position-reward contingency was similar. When a new block started, saccades to the newly rewarded position sped up and anticipatory responses in the contralateral caudate increased to maximum levels after a single trial. By contrast, the speed of saccades to the newly unrewarded position and anticipatory responses in the contralateral caudate decreased more slowly, taking $(\sim 5$ trials to reach stable levels.

Of course, these results establish only correlative, not causal, links between physiology and behavior. Nevertheless, the relationship between the caudate and other oculomotor circuitry provides a tantalizing view of how the anticipatory signals in the caudate might bring about the biased behavioral responses. The authors propose that the spatially selective anticipatory responses in the caudate could be propagated through two inhibitory connections, first to the substantia nigra pars reticulata and then on to the superior colliculus. The end result of anticipatory activity in the caudate would, thus, be to increase responses in a restricted region of the superior colliculus. For example, on a task like the BST that requires a saccade to a target that will appear either to the right or to the left, some caudate neurons on, say, the left side of the brain would respond in anticipation of target onset if rightward saccades are rewarded. This anticipatory activity would, in turn, generate a similar signal in the region of the superior colliculus involved in generating rightward saccades.

The idea that the caudate might be a source of a rewardrelated bias that exerts its influence by increasing the baseline responses of neurons that are involved in generating eye movement is particularly interesting in light of recent theoretical work. For tasks in which the detection, discrimination or identification of a sensory stimulus leads directly to an action (such as an eye or arm movement), accuracy and response times can be accounted for by assuming that the sensory stimulus causes some (noisy) signal in the brain to build up to a threshold value, at which point the action is initiated [1,8,9]. Interestingly, in some cases it might be not only possible, but also in fact optimal, to incorporate psychological factors like reward anticipation and prior probabilities (i.e. uncertainty about the potential outcomes before any stimulus appears) into this framework by simply adding that information to the accumulating sensory signal $[10,11]$. For the BST and similar visually guided saccade tasks, it is tempting to speculate that this quantity, reflecting several sensory and non-sensory factors, is accumulated in oculomotor areas, such as the superior colliculus. According to this idea, the caudate might be just one of several sources that could, in principle, provide information to these oculomotor areas to influence the process of preparing an appropriate eyemovement response on a sensory-motor task.

\section{The advance bias reflects an anticipation of a preferential outcome}

Thus, anticipatory activity in the caudate, corresponding to a spatially selective expectation of reward, could be linked to oculomotor preparatory signals. But which of these factors is central to the function of the caudate? That is, it seems possible that the activity measured in the caudate during the BST did not reflect reward context per se but, rather, a more general anticipation of an impending eye movement. This kind of anticipation is known to be represented in oculomotor areas including the superior colliculus [12,13] and parts of the parietal cortex [14] and is sensitive to target uncertainty: lower the uncertainty and increase the anticipation. To test this idea, Lauwereyns and colleagues used a second task that eliminated target uncertainty by flashing a cue indicating where the saccade target would appear. All correct saccades were rewarded. Recording from a subset of the same caudate neurons that exhibited reward-related activity in the BST, they found that these neurons did not have spatially selective anticipatory responses when target uncertainty was eliminated in this manner. This result seems simply to rule out a general role for the caudate in oculomotor anticipation and to emphasize the importance of reward.

Results from this second task also raise two interesting questions. First, why would a neuron that responds in anticipation of reward associated with a contralateral stimulus in the BST task not also do so in this second task? Indeed, in both tasks, a reward is expected if the contralateral stimulus appears. The answer, it seems, has to do with another form of uncertainty. The second task eliminated uncertainty not only about which eye movement would be made, but also about whether or not there would be a reward. This experiment is similar in spirit to an earlier study that measured spatially selective, rewarddependent anticipatory activity in the caudate using a slightly different saccade-to-target task [7]. Results from that study indicated a similar effect, in which the responses in the caudate were eliminated by eliminating reward contingencies and rewarding on every trial. These results suggest that the caudate could represent an anticipated, spatially selective reward - but only if there is the possibility of an unrewarded alternative as well. That is, the caudate responses might be inherently 'comparative' and, thus, particularly suitable for use in decision-making (even in tasks like the BST, in which certain outcomes might be preferable - even though the subject is never given the opportunity to choose between them!). Exactly how this comparative signal is generated (e.g. are the relative merits actually compared, or is there just an indication that different alternatives are possible?) remains an open question.

The second question raised by these results concerns the selectivity of the responses in the caudate. Of the 41 neurons with anticipatory activity, 31 exhibited spatially selective reward anticipation during the BST. Of these 31, 22 were tested on the second task and most, but not all, 
showed little effects of target uncertainty. An obvious question in studies with such small sample sizes is whether or not the sampled responses reflect the population as a whole. If so, does this imply that there could be small sub-populations of caudate neurons that signal target uncertainty? Or was that selectivity just a chance occurrence? If not, what is the selectivity of most caudate neurons? What other factors affect their responses? Indeed, other response properties have been found in the caudate, including a suppression of responses in anticipation of an eye movement to a rewarded location [7] and a representation of color-reward contingencies [6]. Learning how these different properties are represented across the population of neurons in the caudate will be an essential step in understanding its role in using information related to reward to influence oculomotor behavior.

\section{Conclusions}

The fact that an expectation of reward can strongly bias our decisions and actions - especially, as Paris and I can attest to personally, if the reward involves marrying the most beautiful woman in the world - is not in dispute. What is uncertain is exactly how brain links signals related to reward expectation [3] to the signals responsible for making decisions and preparing actions [15]. Several recent studies have characterized the influence of reward expectation on neural circuits involved in sensory-motor transformations [14,16-20]. The study by Lauwereyns and colleagues [4] adds to that short list and provides novel insights into how the brain might use signals related to a spatially selective anticipation of reward to bias a simple oculomotor behavior. It will be interesting to see whether the same principles can be extended to include other factors and more complex behaviors.

\section{References}

1 Luce, R.D. (1986) Response Times: Their Role in Inferring Elementary Mental Organization, Oxford University Press
2 Green, D.M. and Swets, J.A. (1966) Signal Detection Theory and Psychophysics, Wiley

3 Schultz, W. (2000) Multiple reward signals in the brain. Nat. Rev. Neurosci. 1, 199-207

4 Lauwereyns, J. et al. (2002) A neural correlate of response bias in monkey caudate nucleus. Nature 418, 413-417

5 Hikosaka, O. et al. (2000) Role of the basal ganglia in the control of purposive saccadic eye movements. Physiol. Rev. 80, 953-978

6 Lauwereyns, J. et al. (2002) Feature-based anticipation of cues that predict reward in monkey caudate nucleus. Neuron 33, 463-473

7 Takikawa, Y. et al. (2002) Reward-dependent spatial selectivity of anticipatory activity in monkey caudate neurons. J. Neurophysiol. 87, $508-515$

8 Gold, J.I. and Shadlen, M.N. (2002) Banburismus and the brain: decoding the relationship between sensory stimuli, decisions, and reward. Neuron 36, 299-308

9 Ratcliff, R. and Rouder, J.N. (1998) Modeling response times for twochoice decisions. Psychol. Sci. 9, 347-356

10 Gold, J.I. and Shadlen, M.N. (2001) Neural computations that underlie decisions about sensory stimuli. Trends Cogn. Sci. 5, 10-16

11 Carpenter, R.H. and Williams, M.L. (1995) Neural computation of log likelihood in control of saccadic eye movements. Nature 377, 59-62

12 Basso, M.A. and Wurtz, R.H. (1998) Modulation of neuronal activity in superior colliculus by changes in target probability. J. Neurosci. 18, 7519-7534

13 Dorris, M.C. and Munoz, D.P. (1998) Saccadic probability influences motor preparation signals and time to saccadic initiation. J. Neurosci. 18, 7015-7026

14 Platt, M.L. and Glimcher, P.W. (1999) Neural correlates of decision variables in parietal cortex. Nature 400, 233-238

15 Schall, J.D. (2001) Neural basis of deciding, choosing and acting. Nat. Rev. Neurosci. 2, 33-42

16 Leon, M.I. and Shadlen, M.N. (1999) Effect of expected reward magnitude on the response of neurons in the dorsolateral prefrontal cortex of the macaque. Neuron 24, 415-425

17 Watanabe, M. (1996) Reward expectancy in primate prefrontal neurons. Nature 382, 629-632

18 Hikosaka, K. and Watanabe, M. (2000) Delay activity of orbital and lateral prefrontal neurons of the monkey varying with different rewards. Cereb. Cortex 10, 263-271

19 Tremblay, L. and Schultz, W. (1999) Relative reward preference in primate orbitofrontal cortex. Nature 398, 704-708

20 Tremblay, L. and Schultz, W. (2000) Reward-related neuronal activity during go-nogo task performance in primate orbitofrontal cortex. J. Neurophysiol. 83, 1864-1876

\title{
A prominent role for intrinsic neuronal properties in temporal coding
}

\author{
Jeffrey C. Magee
}

Neuroscience Center, Louisiana State University Health Science Center 2020 Gravier St, New Orleans, LA 70112, USA

\begin{abstract}
A recent report presents evidence that the exact timing of action potential output in rat hippocampal pyramidal neurons is similarly modulated during several diverse forms of behavior. These data suggest that it is, to a large degree, the intrinsic properties of the neurons themselves that produce this temporal coding of information. Thus, this report provides an outstanding
\end{abstract}

example of the importance of single neuronal properties, even during complex behaviors.

Hippocampal theta rhythm is a low frequency $(5-10 \mathrm{~Hz})$ oscillation of extracellular current that results from the collective activity of groups of neurons ([1]; reviewed in Refs [2-4]). This network oscillation occurs during a wide variety of spatial and non-spatial behaviors and can be thought of as a type of timing signal, upon which the action 